\title{
LA ORGANIZACIÓN DEL TRANSPORTE MARÍTIMO EN LA VALENCIA DE LA PRIMERA MITAD DEL SIGLO XV
}

\author{
ENRIQUe CRUSELles \\ Universidad de Valencia
}

La progresiva integración de los mercados europeos y la emergencia de una nueva red de intercambios, surgida como respuesta a la crisis del XIV, conllevó al mismo tiempo una transformación en la organización maritima de Occidente, mediante la difusión de una serie de técnicas e innovaciones que afectaban al mundo del transporte: el retorno a la construcción de embarcaciones de gran tonelaje con capacidad para cargamentos voluminosos y pesados, la difusión de prototipos de naves originarias del mundo maŕtimo atlántico, de la mano de la penetración en el Mediterráneo de las flotas castellana y portuguesa, la irrupción de innovaciones como la combinación de velas cuadradas y latinas o el timón de codaste, junto a la generalización de nuevas técnicas mercantiles que, como el seguro maŕtimo o los fletes discriminativos, implicaban un readaptación mental de los mercaderes y hombres de negocios medievales. En la base de aquellos cambios subyacía la necesidad de superar el tráfico marítimo tradicional basado en la comercialización de mercancías de elevado precio y de asequible coste de transporte. Por ello, los efectos se manifestaban en primer lugar en el precio del servicio. Desde las últimas décadas del siglo XIV y los albores de la siguiente centuria, se concluyó el paso de una estructura rígida de fletes, caracterizada por los precios elevados del servicio de transporte, a otra donde una nueva dinámica en la contratación equilibraba el precio del flete con el valor de la mercancia. Es decir, el coste del transporte de un grupo de mercanclas se distribula de manera distinta entre éstas en relación a su valor de mercado. En una primera fase, esta innovación en el ámbito del transporte era asumida por el gran mercader, único capaz, al 
actuar en una gran escala, de modelar el servicio de transporte según sus exigencias y necesidades. Posteriormente, ya entrados en el XV, hasta los comerciantes con una menor participación en el mercado internacional hicieron uso de esta innovacion técnica. ¿Qué impulsaba esta readaptación técnica? Según la conclusión insuficiente de F. Melis, la causa primaria fue la ambición del mercader por ampliar el repertorio de mercancias y el número de mercados donde actuar ${ }^{1}$.

En un sistema productivo como el feudal, donde la tecnología aplicable al proceso de trabajo era innovada lentamente, la penetración del capital comercial en el proceso productivo era superficial y la aplicación de renovados sistemas organizativos del trabajo era superficial, mayor inversión de capitales por unidad de producción conllevaba reducciones cada vez menos significativas en los costes de producción de la mercancía. Esta tendencia a la estabilidad de los costes, repercutía sobre el margen de beneficios. En aquella época, cuando la competencia entre los especialistas del intercambio aún no habra generado férreos sistemas de monopolio, y en la que la ampliación del número de economías regionales que participaban en las mismas redes comerciales hacía que los efectos de aquella competencia fuesen cada vez más acuciantes, la tendencia decreciente de la cuota de ganancia, solo podía ser contrarrestada, dentro de un marco cultural restrictivo, mediante la aplicación de unas estrategias internas al proceso de circulación. En este sentido se explica la necesidad perentoria de diversificar el espectro merceologico negociado por una misma empresa, en un intento por obtener una mayor cuota de participación en el mercado, a pesar de que los rendimientos decrecientes puntuales de algunas de esas mercancias conllevasen menores beneficios. Esta es una práctica asumida por todas las grandes empresas comerciales de la época. Asimismo, el capital mercantil arbitraría otras estrategias con el mismo fin, siempre dentro de la esfera comercial y de su mundo anexo, el del transporte. Se mejoró el aparato jurídico que rodeaba al contrato, extendiéndose su vigencia a un gran espacio económico, se estructuró una red de sucursales, agentes o corresponsales que facilitaban la circulación de la información, se arbitraron mecanismos de negociación colectiva de capitales, se invirtio en la mejora de los medios de transporte, pasando en muchas ocasiones los

'Federigo MEUS, Werner Sombart e i problemi della nave-gazione nel Medioevo, en Luciana Frangione (ed.), "l trasporti e le comunicazioni nel Medioevow, Firenze, 1984, p. 43. 
mismos mercaderes a ser copropietarios de embarcaciones, lo que permitio un abaratamiento de los servicios, se perfecciono una red financiera a escala interregional, que favoreció la capitalización de las sucursales y agentes así como la circulación de capitales, o se desarrolló un mercado de contratación de seguros marítimos que permitía reducir los riesgos. Dentro de este conjunto de transformaciones, esencialmente empresariales y financieras, se ha de comprender el recurso a la utilización del flete discriminativo como un mecanismo más, desde luego no más importante que otros, con el fin de abaratar los costes del proceso de circulación.

Según indica la documentación notarial, el grupo mercantil valenciano no recurrió a estas estrategias durante las últimas décadas del siglo $\mathrm{XIV}$, si bien el uso del flete discriminativo era conocido en los ambientes comerciales de la ciudad. Los genoveses, la colonia con mayor arraigo, utilizaban este sistema de fijación de precios de transporte para pagar a los patrones y propietarios de navíos de origen valenciano y catalán. En 1367, Guillem Sala, ciudadano de Barcelona, fletaba a Baltasar di Marinis en dos ocasiones su embarcación para transportar mercancías hasta Génova. En el segundo de aquellos contratos, el elenco de precios del transporte según mercancías es más amplio: 6 sueldos por quintal de arroz; 4 sueldos por el de pez; 7 por cada tinaja de trementina; 6 sueldos y medio por quintal de regaliz; un florín y medio por cada bala de paños de lana; y medio florín por cada fardo de seda. En otro flete contratado por Gentil Salvayo con el mismo destino se especificaba el precio de otros artículos, entre ellos la cerámica de Paterna y Manises, que debía pagar a 12 sueldos la tinaja. A finales de ese año, Bernat Mateu y Ramon de Gualba alquilaban a Federico Salvage su lembo para transportar paños hasta Almería, percibiendo 15 sueldos por paño. Años más tarde, en 1375, la coca patroneada por Guerau Roca era arrendada a Antonio di Mari para navegar a Cartagena, donde debia cargar unas mercancias que se transportarian a Genova ${ }^{2}$, diferenciando el precio del servicio según el tipo de artículo: frutos secos, 8 sueldos por espuerta; 15 sueldos por quintal de lana; el anís, a 8 sueldos el quintal; otros ocho sueldos el quintal de cuero.

La documentación notarial del XV ya se hace eco del recurso de los mercaderes locales a estas prácticas. Los ejemplos más antiguos corres-

21367, septiembre 14, octubre 8, noviembre 6 y 23 (ARV, Protocolos, no 2827, R. Pons); 1375, octubre 19 (Idem, no 11215, Desconocido). 
ponden a los viajes comerciales hacia las plazas berberiscas y granadinas, los mercados pañeros y trigueros tradicionales de las empresas valencianas. En 1406 Mateu Turo fletaba su pequeña nave a Jaume Bonet, hijo de Guillem Usall, para navegar hasta Ténès, donde debra cargar trigo con destino a Valencia. En concepto del servicio de transporte el mercader debra pagar siete sueldos y medio por cahiz. Un segundo ejemplo es el alquiler del lembo de Antoni Ruxot realizado por Lluís Eiximeno, mercader de Valencia, a Alf ben Xernit para transportar trigo y otras mercancias desde Mostaganem a Valencia, donde el flete del cereal se fijaba en siete sueldos el cahiz, mientras que el de las otras mercancías en tres sueldos el quintal ${ }^{3}$. La ruta maritima con los mercados y plazas que circundan la Mancha mediterránea parece haber sido la precursora en la adopción de los fletes discriminativos. Con ciertos años de diferencia, esta práctica mercantil se extendió a las otras dos rutas que configuraban la red esencial del comercio interregional valenciano, el tráfico con las islas del Mediterráneo central y el trayecto con los mercados de la Europa atlántica. En el primer caso, el trigo determinaba la elección de precios especiales. En el segundo, la variada tipología de productos agrarios especializados que se exportaban desde el sur del reino, soportaba la parte esencial de los costes del transporte. En 1415 Bernat Roig fletaba su nave a Daniel Barcelo. El trayecto concertado implicaba recalar en Ibiza para cargar sal, que junto a otras mercancias se debía enviar a Palermo y Catania. Con posterioridad, navegaría hacia el sur de la isla para estibar trigo que el patrón debería descargar, siguiendo las indicaciones del mercader o su representante, en Valencia, Orán, Honein, Alcudia o Málaga. En el primer caso, el flete se fijaba en 15 sueldos por salma. Ese mismo año Vicent Colomer alquilaba la barxa castellana del patrón Fernando Alfonso, un sevillano, para transportar mercancias a Cerdeña, en cuyos puertos, Oristano y Cáller, debía cargar trigo que, tras el viaje de retorno, debía estibar en Denia, Gandía o Valencia. El precio venia fijado en relación al puerto de carga: el trigo que se cargase en Oristano, nueve sueldos y medio la salma; diez y medio el de Cáller. De nuevo ese año se concretaba el precio de un fletamiento en relación a las mercancras cargadas en la embarcación, aunque esta vez en la ruta de Flandes ${ }^{4}$.

\footnotetext{
${ }^{3}$ 1406, julio 17 (Idein, no 2408, V. Saera); 1409, febrero 7 (Idem, no 3025). A partir de este momento, los protocolos en que no se cite al notario pertenecen a este escribano de la primera mitad del siglo XV.
}

41415, mayo 28, agosto 16, febrero 27 (Idem, n²416). 
Gregorio Trenta, mercader de Lucca, como conductor de una nave gobernada por Juan Amat de La Coruña, fletaba a Joan Bou y Martl Calbet, éste mercader de Perpignan, la embarcación para transportar productos agrarios cargados en Cullera y Denia al puerto de Brujas. El precio del alquiler se acordaba de la siguiente manera: el arroz pagaba un flete de 11 gros el quintal; la almendra, 14; el anís y el comino, 15; y el vino de moscatel 4 francos y un cuarto la bota.

Por tanto, desde principios del siglo XV, la difusión de fletes discriminativos se practicaba en todas las rutas maritimas utilizadas por los mercaderes valencianos. Ahora bien, durante este perfodo convivieron dos sistemas básicos de contratación de fletes. Por un lado, el discriminativo, que expresado a través de ciertas mercanclas esenciales, hacia recaer sobre éstas los costes del transporte. Por el otro, el precio a tanto alzado, practicado cuando el mercader alquilaba la embarcación a la escarada por un precio global, por lo que en principio no influía el tipo de mercancia estibada. ¿Se trata de la convivencia de dos prácticas mercantiles anacrónicas? Podría ser. Desde luego, no se percibe que una de ellas suponga una superiodad de tipo técnico o mental sobre la otra. Por de pronto, se recurría a ambas para las mismas rutas e idénticas actividades. La tipología de embarcaciones tampoco influye pues encontramos barcas y naves que utilizaban ambos tipos de contratación de precios. Quizás una posible explicación resida en el diferente grado de adaptación de los patrones a los nuevos adelantos técnicos acaecidos en el mundo de la navegación, ya que no es normal que un mismo patrón recurra a ambos sistemas de fijación de los precios. Por su parte, el grupo mercantil valenciano no demuestra un comporta-miento coherente. Jaume Bonet alias Usall, mencionado con anterioridad por el uso del flete discriminativo, pagaba una cantidad fija (135 doblas) al patrón Antoni Moyo por un viaje a Honein y Orán, mientras que años antes su padre, Guillem Usall, reproducía una actitud similar's. Lluis Eximeno contrataba fletamientos tanto a través de un sistema como de otro ${ }^{6}$, de la misma manera que Daniel Barcel $\delta^{7}$, Vicent Colomer ${ }^{8}$, Joan Bonet ${ }^{9}$, Fran-

\footnotetext{
s 1404, julio 31 (Idem, $n^{\circ} 2406$ ); 1405, enero 19 (Idem, n² 2407); 1409, febrero 16 (Idem, n' 3025).

${ }^{6} 1409$, mayo 23 y agosto 7 (Idem, $n^{0} 3025$ ); 1410, diciembre 29 (Idem, $n^{0} 2718$ ); 1411, febrero 9 (Idem, $\left.\mathrm{n}^{\circ} 2412\right) ; 1412$, abril 7 y 13 (Idem, $\mathrm{n}^{\circ} 2413$ ).

${ }^{7} 1413$, abril 17 (Idem, $n^{0} 2414$ ); 1415, mayo 28 (Idem, $n^{0} 2416$ ); 1416, enero 31 y junio 20 (Idem, $n^{\circ}$ 2417).
} 
cesc Pellicer ${ }^{10}$ o Joan Eiximenis ${ }^{11}$. Este doble uso de prácticas mercantiles diferentes, puede comprenderse mejor si se acepta una relativa difusion de otras técnicas y de una nueva mentalidad financiera. F. Melis explicaba una segunda fase en la consecución de la revolución de los fletes a través del perfeccionamiento contable: "...la prima manifestazione di costo della navigazione che egli incontrava era quella unitaria, complessiva -alla liquidazione del prezzo del noleggio totale-, per una congerie di beni anonimi, i quali, quando se ne predisponeva la distribuzione, riacquistavano la loro individualità; e il mercante scomponeva quel costo, in funzione di quattro variabili: secondo la situazione di mercato, la categoria delle merci da collocarvi (soprattutto per il loro prezzo), i quantitativi e il risultato economico d'insieme..." 12 .

Bajo esta concepción, es lo mismo que el precio se exprese a través de una cantidad global que el método de discriminación de precios según mercancias. Son manifestaciones de una misma estrategia financiera. Claro está, eso significa que la innovación en los métodos contables habra sido asumida y era utilizada por el grupo mercantil local. Presuponer este adelanto técnico, dada la escasa supervivencia de libros contabilidad en los libros valencianos, es cerrar en falso el problema. Respecto a este tema, tenemos un pequeño indicio. Se trata de un cuadernillo de cuentas escriturado por el mercader valenciano Andreu Conill entre 1420 y 1421, años en los que permaneció en las ciudades de Almería y Málaga ${ }^{13}$. En ese cuadernillo Conill reflejaba los costes de la comercialización de diversas mer-

\footnotetext{
'1415, agosto 16 (Idem, $\mathrm{n}^{0} 2416$ ); 1416, enero 4 (Idem, $\mathrm{n}^{0} 2417$ ); 1417, octubre 15 (Idem, $\mathrm{n}^{\circ} 2418$ ); 1418, septiembre 24 (Idem, $\mathrm{n}^{\circ} 2419$ ).

${ }^{9} 1417$, septiembre 2 y 30, diciembre 29 (Idem, $\mathrm{n}^{0} 2418$ ).

${ }^{10} 1416$, junio 25 (Idem, $n^{0} 2417$ ); 1418, octubre 6 (Idem, $n^{0} 2419$ ).

"1416, junio 25 (2417); 1419, agosto 28 (Idem, n 2420).

${ }^{12}$ Federico MEUS, ob. cit., p. 44. A este respecto, es importante tener presente que a pesar de que la colonia italiana presente en la ciudad de Valencia suela recurrir a la expresión del flete discriminativo, también se encuentran casos de operadores extranjeros que utilizan ambas prácticas. Es el caso de algunos de los operadores económicos extranjeros más importantes de la ciudad: el marquesano Francesco di Nofre di Fabriano (1437, mayo 22. ARV, Protocolos, $n^{\circ}$ 2774), los florentinos Clemente di Sumaya (1437, junio 27. Ibidem; 1440, marzo 4 y mayo 27. Idem, no 4391), Boxo di Giovanni (1440, junio 6. Ibidem), Jacobo degli Strozzi (1440, marzo 10. Ibidem) o Veri di Bardi (1425, julio 27. Idem, $n^{\circ} 2423$ ), el pisano Niccold Torrizani (1438, mayo 15. Idem, no 2434), los lombardos Antonio di Servano (1436, febrero 28. Idem, $n^{0}$ 2433) o Jacobo Danya (1441, julio 6. Idem, $n^{\circ} 2411$ ), o el genovés Foderico di Nigro (1420, abril 25. Idem, no 2421).

${ }^{13}$ ARV, Varia, Libros, no 309.
} 
cancías (paños valencianos, merluza, seda, cera, etc.) desglosados según el tipo de producto, trasladando el coste total de cada apartado a un libro mayor. Esta práctica contable, donde los costes se podian contrastar con los precios obtenidos en la venta de las mercancías, permitirfa evidentemente desglosar los costes unitarios de la mercancía, incluyendo en ellos los obtenidos en el proceso de circulación. Si consideramos que esta práctica contable realizada por Andreu Conill (del cual no hemos tenido confirmación de que fuese un mercader de remarcada potencialidad financiera y organizativa) era general al medio mercantil valenciano, la fijación de un precio del servicio del transporte a tanto alzado significaba de todos modos, como explicaba F. Melis, la discriminación del coste de transporte de las mercancías según las características particulares de estas últimas. Práctica que permitf́a la asunción de un mayor porcentaje del coste por las mercanć́as más valiosas y una ampliación del espectro merceológico comerciado. Dentro de este proceso, el reflejo en algunos contratos de fletes de los costes particularizados según mercancías, era la expresión última de esta evolución. La empresa valenciana, imitando a la italiana, había conseguido controlar y supeditar el acto del transporte a la actividad mercantil. En 1409 Antoni Ruxot fletó a Lluís Eximeno su embarcación por un precio fijo, 145 doblas, precio que incluía el transporte del viaje de ida y de retorno a Mostaganem. Sin embargo, el mercader a su vez alquilaba la embarcación a otros dos individuos, un escudero para transportar hombres en el viaje de ida y un mercader mudéjar con el que compartía el cargamento de trigo en el viaje de retorno. En ambas ocasiones, el precio variaba según la mercancia transportada ${ }^{14}$.

Un argumento más puede darnos a entender que la nueva técnica se habra difundido en Valencia ya en esta época. A principios de 1418, Zanobio de Bartolo, un comerciante florentino fletó «a la escarada» la nave de Francesc Giner, un ciudadano de Barcelona. El flete global se fijaba en 1100 ducados de oro venecianos. Dias más tarde, denominando como conductor de la nave, el florentino fletaba a Nicolau Montell, un comerciante valenciano de origen italiano, dicha nave para transportar 500 quintales de mercancias hasta Siracusa y Venecia. El precio del servicio se explicitaba en el caso del arroz y de la lana, mientras que para las otras mercancías serfa Francesc Joan de Arezio, un corredor de la ciudad, el que fijarla el

\footnotetext{
${ }^{14} 1409$, febrero 7, enero 30 y febrero 7 (ARV, Protocolos, no 3025).
} 
flete de su transporte ${ }^{15}$. Esto puede dar a entender que el mercado valenciano gozaba ya de unas estructuras técnicas y organizativas -aún en estado de formacion- que permitian precisar los costes del transporte que debran asumir las mercancías, según sus calidades y valores de mercado.

En cualquier caso, los elementos mencionados (el cambio en el sistema de contratación de los fletes, transformaciones de la industria naviera y el aumento de las flotas) no fueron suficientes en la innovación sustancial del sistema de comercio maritimo. La empresa valenciana siguio asiéndose a unas estrategias financieras y organizativas que manifestaban su naturalesa minimalista. Ante todo persistra en el ambiente mercantil local, a pesar del desarrollo del mercado de seguros marítimos y de la variación del sistema de contratación de los fletes, una resistencia a la concentración de capitales. Rastreamos esta actitud incluso en las actas de constitución de compañas. En 1416, Antoni Garriga y Francesc Siurana, el cambista, formaron una sociedad con fines mercantiles con Ramon Puigroig y Pere Corbi ${ }^{16}$. Por medio de esta asociación se mancomunaban un total de 3300 libras, las cuales invertidas en mercancias serfan gestionadas por los dos últimos en la tienda que poselan junto a la vieja lonja de la ciudad. En el ámbito del comercio local la actividad de los socios administradores no se vera mediatizada en modo alguno. Sin embargo, fuera del ambiente local, donde la estructura empresarial demostraba sus incapacidades e inadaptaciones, nadie quería arriesgar. Este miedo latente se refleja en los acuerdos tomados: "Item, és convengut entre ells que los dits En Ramon de Ruigroig e En Pere Corbl puxen arrischar de bens, robes e peccúnies de la dita companya a risch, perill e fortuna de tots los dits companyons per mar e 0 per terra e o per aygua dolç tro en quantitat de dohentes lliures reals de Valencia. Açd declarat que cobrades les robes e mercaderies en les quals se.s merçaran les dites CC lliures que los dits En Ramon e En Pere puxen aquells smerçar e arrischar tantes e quantes vegades a ells plaurà. Axi emperò entés que tots temps correguen a risch de la dita companya dohentes lliures e no pus". Un ejemplo similar es el de la compañfa mercantil formada por Pere y Guerau Bou, Jaume Guils y Guerau Bellviure en 1422, con un capital social de 3000 libras ${ }^{17}$. Pero con una diferencia: asi como

\footnotetext{
15 1418, febrero 5 y 12 (Idem, no 2419).

161416, enero 8 (Idem, $n^{0} 2417$ ).

${ }^{17} 1422$, junio 23 (Idem, $n^{\circ} 2418$ ).
} 
el centro mercantil de la anterior compañía residía en la ciudad de Valencia, esta otra se constitura en base a una organización bicéfala en torno a dos casas, una en Valencia y la otra en Génova. La preocupación de este otro grupo de comerciantes segula girando en torno a la libre capacidad de los socios para gestionar el capital mancomunado. Entre la formulación restrictiva que surge de estos miedos subyacentes destaca la referente a la limitación de la cantidad de bienes comerciados: "Item que nengú qui administrarà les dites cases no trameta en les fustes que carregarà robes per qualsevulla de les cases sinó en quantitat de cinchcentes lliures barchinonenses; e quant que fos menys no sia més, que si més hi metrà sia a risch de aquell qui carregarà les més quantitats hon assegure lo més".

El temor al riesgo implícito en la actividad maritima, el miedo que anida en los límites y resquicios de un mundo mal conocido, donde estos hombres no debran moverse a gusto, opera como barrera infranqueable en el sistema de reproducción de capitales. Se trata pues de obtener ganancias de la actividad mercantil, pero ante todo de no sufrir pérdidas. Esta necesidad de limitar los riesgos, que actúa como mecanismo ralentizador de la dinámica comercial, se convierte en un viejo fantasma del ideario cultural del mercader local. Tener presente esta actitud mental ayuda a comprender la actividad mercantil y maritima de las empresas valencianas. Un comerciante local asume al año pocos negocios fuera de su mercado natural. $Y$ cuando negocia procura no asumir riesgos: por un lado, diversifica al máximo el tipo de mercancías comerciadas, evitando ası posibles respuestas negativas de los mercados; por el otro, fragmenta el volumen comerciado de cada mercancía. El 7 de abril de 1412, Lluís Eximeno fletaba la barxa castellana de Joan Mora para trasladarse a Ténès donde debra cargar trigo, con el que regresarla hasta Valencia. Dlas más tarde volvía a fletar otra barxie, ésta gobernada por Joan Pons, para realizar un trayecto parecido con fines similares: navegar hasta los cabos de Cervera y de La Mata, para cargar sal, continuar hasta Honein y, de alli, a Ténès, donde debla cargar trigo que llevaría hasta Valencia. Comportamiento similar desarrollaba el comerciante Joan Armenguer. En 1417 nombraba un procurador para que se trasladase hasta Palermo y recuperase un cargamento de lana que el patrón, Pedro Diez de Bilbao, de manera descuidada habla abandonado allr, cuando tenía que haberlo transportado hasta Venecia. Dias más tarde, un comerciante florentino reconocía que Armenguer tenfa la propiedad de 160 sacos de lana que habla cargado ya en la nave de Polo Blanco, con destino a Venecia. En 1420, en el plazo de nueve días, Armenguer fletó dos em- 
barcaciones distintas, la coca de Francesc Rispau y la nave de Pedro Ferrández, para realizar el mismo trayecto. Se trataba de navegar desde Valencia hasta Alcudia, vender mercancías y comprar dátiles, que se transportarían hasta el puerto de Cádiz. En agosto de 1447 Gabriel Andreu se hacía asegurar pequeños cargamentos de similares características transportados al mismo puerto, Sevilla, por dos embarcaciones distintas ${ }^{18}$.

En otros casos esta fragmentación del negocio entra dentro de una estrategia global de las compañas por conjugar la disminución de riesgos con la ampliación de la cuota de representación en el mercado extranjero. En 1439 Galceran Martí fleto dos embarcaciones en el plazo de cuatro días. La primera, un bergantín gobernado por Bernat Valls, debía realizar directamente sin escalas el trayecto entre Valencia y Orán. El segundo, otro bergantín, guiado esta vez por Lluís Claver, se dirigirfa directamente a la playa de Mostaganem, cercana a la ciudad de Orán, donde realizaría las labores de estibaje para retornar nuevamente a Valencia. En el verano de 1440 nuevamente Galceran Martí fletaba otra embarcación, un ballener sevillano con un arqueo aproximado de 900 quintales para navegar de nuevo hasta Orán. En un plazo inferior a un mes, volvfa a fletar nuevamente esa embarcación para realizar el mismo trayecto ${ }^{19}$. Este sistema de comercialización restricivo parece instalarse en los hábitos de comportamiento de las compañras mercantiles locales.

La tendencia a la fragmentación de los cargamentos se manifiesta asimismo en la inferioridad, a nivel cuantitativo, de los contratos de fletamiento a tanto alzado de todo un buque, frente a la contratación del transporte de una limitada cantidad de mercancías fijada a través de una póliza de embarque. A pesar de que el arqueo de las embarcaciones fuese en aumento durante este periodo, la capacidad de transporte de los navíos segula superando la potencialidad operativa de las empresas mercantiles. Por ello, es normal encontrar casos en que el navio es alquilado de manera colectiva por un grupo de mercaderes. En 1415 Berenguer Ameller flet 6 su lembo a tres comerciantes valencianos, Francesc Monfort, Pere Monfort y Jaume Eximeno que corrfan conjuntamente con la responsabilidad de pagar el servicio. Del mismo modo, Antoni Ruxot alquilo su nave a Francesc

\footnotetext{
181412 , abril 7 y 13 (Idem, $n^{0}$ 2413); 1417, enero 11 y 21 (Idem, $n^{0} 2418$ ); 1420, abril 16 y 25 (Idem, n' 2421); 1447, agosto 29 (APPV., protocolo n' 25974, P. Castellar).

191439, eeptiembre 11 y 15 (ARV, Protocolos, n' 2435); 1440, agosto 17 y septiembre 14 (Idem, $\mathrm{n}^{\circ}$ 4391).
} 
Monfort, Joan Guilla, Joan Valls y Gabriel Pomar. En 1418, Joan Ferrer arrendaba su nave a tres comerciantes, diviendo la participación en mitades, una para Galceran Marti, y otra, para Joan Franch y Francesc d'Artés. También Pere Cerdà fletó su galeota de 17 bancos en 1438 a varios mercaderes: Bernat Sallit, Galceran Martf y Pere Baldovi, los cuales como conductores dicte galiote trabajaban junto al patron en obtener el dinero suficiente para armar y avituallar la embarcación ${ }^{20}$. Se trata de casos extremos, por el elevado número de mercaderes participantes en cada contrato. Sin embargo, es también normal que la embarcación, sobre todo si es de un tonelaje considerable, sea alquilada por dos mercaderes ${ }^{21}$.

La versatilidad del sistema de transporte es mucho más amplia. La contratación de toda una nave por un comerciante no significa que éste comerciase sólo productos suyos, sino que, a partir de ese momento, implicaba la organización del acto de transporte dentro de la estrategia comercial y financiera de su empresa. Para ello, el mercader, que habla adquirido el flete de la embarcación, podía llegar a un acuerdo con el patrón sobre el reparto del precio del transporte de las mercancias de otros comerciantes, asumir por cuenta propia el alquiler a otras personas o trabajar como comanditario y factor de mercaderes de su ciudad en los mercados extranjeros hacia los que llevaría sus mercancías. Cuando a principios de 1409 Lluís Eximeno fletó a la escarada el lembo de Antoni Ruxot, con una capacidad de 400 cafices, para realizar un viaje a Mostaganem, no lo hizo sólo pensando en un negocio propio. Para el viaje de ida Eximeno habra fletado a su vez la embarcación de Ruxot a un escudero de Denia, Nicolau de Séntia, para que en el viaje de ida transportase a 25 hombres. Para el viaje de retorno, fletaba a All ben Xernit el navio para transportar 200 cahices de trigo, la mitad de la capacidad de la embarcación, o bien, dentro de ese límite, cera, cueros y glasa et non alias mercaturias. Si el precio del servicio contratado por Eiximeno se cifraba de manera global, en 145 doblas

\footnotetext{
${ }^{20} 1415$, abril 30 (Idem, $n^{\circ} 2416$ ); 1416, marzo 24 (Idem, $n^{\circ} 2417$ ); 1418, febrero 17 (Idem, n' 2419); 1438, mayo 12 (2), 21 y 23 (Idem, n' 2434).

${ }^{21}$ Citaremos, sin desarrollar, algunas referencias: 1408, agosto 20 (Idem, $n^{0} 2410$ ); 1409, febrero 7 (Idem, $n^{0} 3025$ ); 1411, febrero 9 (Idem, $\left.n^{0} 2412\right) ; 1413$, abril 17, noviembre 6 (Idem, $\left.n^{0} 2414\right) ; 1415$, febrero 27 (Idem, $\left.n^{0} 2416\right) ; 1416$, junio 20 y 25 (Idem, $n^{0} 2417$ ); 1417, septiembre 2 (Idem, $n^{\circ} 2418$ ); 1418, mayo 2 y octubre 26 (Idem, $n^{\circ} 2419$ ); 1421, mayo 2 (Idem, $\left.n^{0} 2726\right) ; 1428$, enero 9 (Idem, $n^{0} 505$, G. Cardona); 1432, febrero 11 (Idem, $n^{0}$ 2429); 1433, junio 10 y 27 (Idem, $n^{\circ} 2733$ ); 1435, mayo 16 (Idem, $n^{\circ} 2432$ ); 1440, mayo 9 (Idem, $\mathrm{n}^{\circ} 4391$ ); 1441, febrero 22, junio 16, julio 14 y agosto $3\left(\right.$ Idem, $\mathrm{n}^{\circ} 2411$ ).
} 
"tam pro nolito dicti frumenti quam dictarum aliarum res...tam de intrata quam de exita", el realquiler realizado por el mercader se ajustaba al valor de las mercancías: dos doblas y media por hombre transportado, 7 sueldos por cahiz de trigo y 3 sueldos por quintal de otra mercancía. Si tenemos presente que en estas fechas, y como especifican los contratos, la dobla cotizaba a 18 sueldos, y suponemos que el mercader mudéjar cargase los 200 cahices de trigo y no de otra mercancía, Eximeno, por ambos subárrendamientos, obtenía algo más 140 doblas, es decir, casi el flete que debra pagar al patrón. Luego su mercancía transportada casi no originaba costes de circulación ${ }^{22}$. De todo esto se deriva que, a pesar de que la documentación nos presente un sistema más o menos privatizado de transporte, la realidad es muy diversa. Viajar por mar, comerciar mercancías por medio de embarcaciones, es una actividad básicamente colectiva, que pone diariamente a prueba los lazos de solidaridad del grupo mercantil local. Cohesión que tiene siempre como móvil económico la reducción de los costes del transporte.

A principios de septiembre de 1417 el barcelonés Francesc Giner fletaba su nave a Joan Bonet y Joan Llobera, para un viaje que, tras recalar en Ibiza, Mallorca y Cáller, tenfa por principal función transportar trigo desde los cargadores de Termini y Agrigento a Valencia. Días más tarde ambos mercaderes formaban una sociedad mercantil de claro matiz familiar, puesto que intervenían los hermanos de Joan Bonet, con un capital social de 1500 florines, dinero que se invertiría en las mercancías que debra transportar la nave de Giner ${ }^{23}$. La participación colectiva era más común en las grandes embarcaciones, cuyo arqueo difícilmente podfa ser completado por una única empresa. A finales de 1418 Martín Pérez de Gamboa, vizcaíno, señor y patrón de una nave de gran tonelaje, fletó la embarcación a Francesc Pellicer para realizar un largo trayecto que, tras recalar en Jávea, Mesina, Candía, Rodas, llegaría a Quíos para retornar nuevamente a Valencia, permitiendo incluso la posibilidad de continuar camino hasta Flandes. Como este fletamiento resultaba demasiado caro para un solo mercader, se debia buscar la manera de afrontar el pago de los 1400 florines del flete. El único modo era buscarse acompañantes en el viaje. En esos días Pellicer realizó varios contratos de comandas en los que el mer-

\footnotetext{
${ }^{22} 1409$, enero 30 y febrero 7 (2) (Idem, $\left.n^{0} 3025\right)$.

${ }^{23} 1417$, septiembre 2 y 16 (Idem, $\left.n^{0} 2418\right)$.
} 
cader se obligaba a llevar hasta Levante diversas mercancías de otros importantes comerciantes de la ciudad: Francesc Siurana, por valor de 1050 florines, y Francesc Aragonés, por 100 florines. Al mismo tiempo, otro comerciante, Joan Valero, se constituía como socio comanditario de Lluís Gaço, del que recibla un pequeño cargamento de paños. $Y$ en ese mismo viaje, la viuda de Antoni Garrigues hacía asegurar varios fardos más con paños ${ }^{24}$.

En el caso de los grandes navíos que realizan el trayecto marcado por las grandes líneas del sistema mercantil internacional, la heterogeneidad de los cargamentos es más acusada. La galera gobernada por Martí Pardo de la Casta recalo en Valencia en 1436. Aqui cargo, entre otras, mercanclas propiedad de Llorens Soler, Bonanat Bellpuig y Galceran Dalmau. Siempre pequeños cargamentos: de Soler, cuatro balas de paños, dos aludas de azafrán, tres cargas de arroz y un pequeño costal de nuez moscada; de Bellpuig, seis balas de paños, un costal de polvo de clavo, un fardo de oropimiente y 69 costales de arroz; de Dalmau, 25 cargas de arroz y 38 costales de quesos sardos. El trayecto, tras el pertinente desvío a Ibiza para cargar sal, segula costeando el reino de Valencia, para recalar después en Almería, Almuñécar, Málaga y, tras llegar a Alcudia, retornar a Valencia. Asimismo, los comerciantes aseguraban las mercancias que eran cargadas con destino a Valencia. La galera grossa de Barcelona patroneada por Pere Joan de Sant Climent recalo en la primavera de 1437, en uno de sus habituales viajes a Berbería y Flandes. En aquella ocasión varios mercaderes valencianos utilizaron la nave para transportar bultos y fardos hasta el norte de Africa, asl como recurrieron a ella en el viaje de retorno. Entre esos mercaderes estaban Daniel Barcelo, con dos fardos de seda, Bernat Penyarotja, con otro fardo, Vicent Granulles, con un fardo más y las mercancias que se obtuviesen en Túnez de su venta. Para el viaje de retorno se contaba con los artículos comprados por Francesc Pons, Joan Penya-rotja y otros corresponsales suyos, o los de Bertomeu Ros, junior. La galeota del mallorquín Pere Cerdà partió antes del verano de 1438 hacia los puertos del reino de Granada y de Berberfa. En aquella embarcación se había cargado

\footnotetext{
${ }^{24} 1418$, octubre $6,7,13$ y 21, noviembre 4 (Idem, $n^{0} 2419$ ). Con pequeñas embarcaciones, el mercader conduhidor no necesitaba realizar muchos contratos en comandita para aubvenir a la financiación del viaje. Así, Vicent Colomer, que había fletado una barxie castellana a Femando Alfonso para navegar hasta Oristano y Cáller, se convirtió en socio comanditario de Joan Mainé, del cual debía vender un cargamento de paños, invirtiendo el capital en trigo (1415, agosto 16 y 20. Idem, $n^{0} 2416$ ).
} 
20 paños propiedad de Joan Sallit, que comerciaba su hermano Bernat, tres balas de paños de Mateu de Celis, quince balas de paños valencianos cargados por Estefano di Rabis y Lança de Cella, comerciantes lombardos y, en el viaje de retorno, las mercanclas propiedad de Francesc Pons cargadas por Miquel Penya-rotja y Jaume Navarro en Almería ${ }^{25}$. Embarcaciones de tamaño mediano o grande, todas de diferentes naciones, atlánticas y mediterráneas, recalaban en Valencia durante breves períodos de tiempo para completar sus cargamentos. En algunos casos, lo hacían con regularidad. Viajar la mayor parte del trayecto con la bodega rellena y la cubierta ocupada, significaba no sólo beneficios para el patrón y los propietarios del navío, sino una reducción de costes para los mercaderes que en primera instancia habran fletado la embarcación. Como decía F. Braudel, las naves del gran comercio internacional, y por derivación todas, cada una en su medida, eran verdaderos «bazares ambulantes ${ }^{26}$.

A pesar de que la adopción de ciertas técnicas pudiese significar un aumento de la productividad, hecho difícilmente demostrable con las fuentes disponibles en la actualidad ${ }^{27}$, la realidad es que los marinos de finales de la Edad Media siguieron practicando el mismo arte de navegación, basado en la observación del perfil de las costas que servían de referencia para seguir la derrota: "Il n'y eut donc pas de navigation astronomique en Méditerranée avant le XVII siècle, car, vu les moyens techniques et scientifiques de l'époque, elle eut été moins rigoureuse que l'estime à vue de côte ou sur de petits parcours, telle que se pratiquait la navigation en Médi-

\footnotetext{
${ }^{25} 1436$, mayo 25, 26, junio 1 y 21 (Idem, $n^{0} 2433$ ); 1437, mayo 7 (2) y 11 , junio 1 y septiembre 14 (Idem, $\mathrm{n}^{0} 2774$ ); 1438, mayo 27, 28, 29 y 31, junio 5 (Idem, $\mathrm{n}^{0} 2434$ ).

${ }^{26}$ Se trata de un aspecto compartido por todas las economías mercantiles de la época. Así, por ejemplo, una coca veneciana que fue atacada por piratas genoveses frente a Cádiz, reproduce la misma imagen. Se trataría de una embarcación no superior a las 400 botas en la que la lista de mercaderes afectados por el robo ascendía a 34 (Philippe BRAUNSTEIN, La capture d'une coque vénitienne sur la route de Flandre au début du XV siècle, en «Horizons marins. Itinéraires spirituels (V*-XVIIF) $)_{\infty}$, II, Paris, 1987, pp. 123-135.

${ }^{27}$ Frederic C. LANE, Le navi di Venezia fra i secoli XIII e XVI, Torino, 1983, pp. 201 226. Los escasos datos utilizados desde el periodo altomedieval al siglo XVI, parecen indicar más bien que los avances. tecnológicos no significaron en ningún modo un aumento de la productividad marítima, entendida como reducción de los tiempos de navegación (Abraham $\mathrm{L}$. UDOVITCH, Time, the sea and society: duration of commercial voyages on the southern shores of the Mediterranean during the High Middle Ages, en aLa navigazione mediterranea nell'Alto Medioevo», II, Spoleto, 1978, pp. 508-509).
} 
terranée et jusqu'à la mer du Nord"28. El método de estima en la navegación costera no es la única razón. Otros historiadores han esgrimido nuevas causas para explicar la perduración de la navegación de cabotaje: "La larga duración de los viajes..., los peligros, la necesidad de aprovisionarse de agua y de tener noticias sobre la situación política de ciertas zonas marítimas o continentales donde la paz era precaria, obligaban a hacer escalas técnicas y paradas en determinados puertos"29. Pero, ante todo, el ritmo y los rumbos de las embarcaciones medianas y de los grandes navios estaban mediatizados por la necesidad de visitar todos los mercados posibles, los cuales debran prospectar. Es tanto el caso de Venecia ${ }^{30}$ como el de Génova: "Les voyages génois «per costeriam» impliquent au contraire de nombreaux arrêts, une sorte d'éparpillement des activités, jusqu'en de médiocres escales, accidentelles et liees aux occasions; escales dont on tait meme le nom mais que la formule générale «faire la côte» recouvre" ${ }^{31}$. Navegación por escalas que en el caso catalán de la «línea de Levante», implicaba la dilatación de los viajes: "Cal, doncs, concloure que la lentitud general dels transports catalans, compartida per galeres i per naus, es devia al gran nombre de les escales tocades, especialment si es té en compte que en l'únic itinerari de Llevant s'incloïen sovint també les escales de Romania o les de Barbaria, a més, naturalment, de les de Sicnia"32. También en el caso de los viajes de las galeras que, atracando en Mallorca, llevaban hasta Flandes se confirma el mismo hecho: "La navegación, en el siglo XIV, solía ser principalmente de cabotaje, no alejándose excesivamente de la costa, por tener en ella un apoyo"33.

La navegación mediterránea ( $\mathrm{y}$, por derivación, en esta fecha, antes de la necesidad de adentrarse en el oceáno, aún europea) era de cabotaje:

\footnotetext{
${ }^{28}$ Avelino TEXXERA DA MOTA, L'art de naviguer en Méditerranée du XIIF au XVIIF siècle et la création de la navegation astronomique dans les océans, en aLe navire et l'Economie Maritime du Moyen Age au XVIII', principalment en Mediterranéex, Paris, 1958, p. 130.

${ }^{29}$ Michel MOLAT DU Jourdin, Europa y el mar, Barcelona, 1993, p. 68.

${ }^{30}$ Alberto TENENTI Corrado VIVANTI, Le film d'un grand système de navigation: les galères marchandes vénitiennes. XIV-XVF siecles, «Annales E.S.C.», 1961, I, pp. 83-86.

31Jacques HEERS, Gênes et l'Afrique vers 1450: les. voyages uper costeriam», "Anuario de Estudios Medievales», 21 (1991), p. 234.

${ }^{32}$ Mario DEL. TREPPO, Els mercaders catalans i l'expansió de la Corona catalano-aragonesa al segle $X V$, Barcelona, 1976, p. 63.

${ }^{33}$ Francisco SEVILLANo COLOM, De Venecia a Flandes (via Mallorca y Portugal, siglo XIV), «Boletín de la Sociedad Arqueológica Luliana», XXXIII (1968), pp. 25-26.
} 
"En esta época [se refiere al XVI], navegar equivale, sobre poco más o menos, a seguir la costa, como en los albores de la marinería. Es «saltar de roca en roca, como los cangrejos ${ }^{. . .}{ }^{34}$. Por lo menos, hasta la aplicación de las máquinas de vapor en la marina. Hasta cierto punto, la dialéctica entre navegación de cabotaje y grandes líneas de navegación es ficticia. En el cuarto tomo de la obra compilada, tras la Pratica della mercatura de Giovanni di Antonio da Uzzano ${ }^{35}$, escrita en 1442, en el segundo libro se halla el Compasso a mostrare a navicare dall'uno stretto all'altro, una especie de libro de derrotas donde se hace una breve descripción de las costas con referencia a las distancias entre los lugares de atraque. Asi se expresa el subtítulo: "Qui si comincia lo compasso per mostrare a navicare per tutte le Terre marine, e Isole, e mostrati quante miglia è da l'una Terra all'altra, e dall'uno Porto all'altro, e dall'un'Isola all'altra...". Constantes menciones a la necesidad de conocer la costa, de aprender pues, a costear. Suponemos que la adición de esta parte a la Pratica della mercatura tenía por objeto ofrecer a los operadores una serie de conocimientos mínimos sobre la navegación mediterránea ${ }^{36}$. Aún en los derroteros españoles de mediados del presente siglo, la Marina española segura editando el perfil de las costas del Mediterráneo y explicando a los patrones de veleros, según la época del año y los vientos dominantes, los rumbos a seguir, tomando como referencias de las derrotas elementos característicos de aquellas mismas costas ${ }^{37}$.

Eso no implica que en algunas circunstancias especfficas se abandonase la práctica de la navegación de cabotaje y los barcos se adentrasen en el mar, realizando el trayecto entre dos puertos directamente. En un mundo comercial tan competitivo como el Mediterráneo, como recuerda M. Mollat, y especialmente con las embarcaciones de mediano calado, la necesi-

\footnotetext{
${ }^{34}$ Femand BRAUDEL, El Mediterráneo y el mundo mediterráneo en la época de Felipe II, I, México, 1987, p. 133.

${ }^{35}$ Gio. Francesco PAGNINI DEL VENTURA, Della decima e di varie altre gravezze imposte dal comune di Firenze, della moneta e della mercatura de' fiorentini fino al secolo XVI, Lisboa-Lucca, 1766, (ed. fac., Bolonia, 1967).

${ }^{36}$ Esta necesidad de conocer el sistema de navegación común también se demuestra por la aparición de cartas de navegación entre los inventarios de bienes de los mercaderes barceloneses (Claude CARRERE, Barcelona 1380-1462. Un centre econòmic en epoca de crisi, I, Barcelona, 1977, p. 208).

${ }^{37}$ Derrotero de las Costas del Mediterráneo, Cádiz, 1950.
} 
dad de adelantarse a la competencia, favorecía los viajes directos ${ }^{38}$. Así, en los fletamientos que realiza la familia Usall a principios del $\mathrm{XV}$, los viajes a la costa africana, por otra parte no muy distante, son directos, sin que se prevea escalas ante del retorno. Los contratados por Guillem Usall en 1404 y 1405 con dos llauts distintos fijaba un viaje sin escalas a Honein, donde se prevela recalar durante diez y doce dlas seguidos, para retornar a Valencia. La nave alquilada por Jaume Bonet alias Usall, deberfa hacer escala en Denia, tras lo cual navegaría directamente hasta Ténès, donde permanecería atracada quince días. La barca de Berenguer Armengol fletada por Galceran Martl, tras recalar un día en Gandía, navegaría recta via a Mostaganem, donde atracaría durante diez días, tras lo cual regresaría a Valencia ${ }^{39}$. De idéntica manera, embarcaciones fletadas durante esas décadas por Martr, individualmente o junto a compañeros, realizarían el mismo recorrido, o a otros puertos norteafricanos próximos, teniendo en ocasiones por única escala el puerto de Denia ${ }^{40}$. Ejemplos similares encontramos en otras partes del Mediterráneo. Y todos ellos nos lleva a una misma conclusión: conforme se perfecciona la red de operadores economicos permanentes en los mercados extranjeros, es menos necesaria la presencia prolongada de las naves con las mercancias enviadas por el jefe o la casa madre en los puertos que marcan el itinerario. En cierto modo, el corresponsal, el agente comercial, sustituye los periplos marítimos. Aunque en la actualidad es difícil reconstruir la actividad de unos hombres que han dejado escasa huella en la historia, podemos hacernos una idea siguiendo los movimientos de Andreu Conill, mercader del que ya hemos hablado páginas atrás. Hacia el 22 de octubre de 1420 la nave de Bernat Bru arribaba a Almería, descargando 21 balas de paños valencianos de diferentes calidades y una mula que Francesc Marti enviaba a Conill. Tras tentar el mercado de aquella ciudad, Conill partía a finales de ese mes hacia Málaga. Un viaje realizado por tierra con una recua de mulas que duró nueve días a causa del mal tiempo, que le obligo a guarnercerse en Motril y caus 6 algunos desperfectos. A lo

\footnotetext{
${ }^{38}$ Michel MOLLAT DU JOURDIN, ob. cit., p. 101.

${ }^{39} 1404$, julio 31 (ARV, Protocolos, $n^{0} 2406$ ); 1405, enero 19 (Idem, no 2407); 1406, julio 17 (Idem, no 2408); 1434, julio 5 (Idem, $n^{\circ} 2431$ ).

${ }^{40} 1418$, febrero 17, mayo 2 (Idem, $n^{0} 2419$ ); 1437, julio 11, septiembre 24 (Idem, $\mathrm{n}^{0}$ 2774); 1439, enero 10, mayo 16, septiembre 11, is (Idem, $n^{\circ} 2435$ ); 1440, enero 23, agosto 17, septiembre 14 (Idem, no 4391); 1441, septiembre I (Idem, no 2411); 1443, marzo 13 (Idem, $n^{\circ}$ 796, M. Doto).
} 
largo de noviembre vendió parte del cargamento de paños y a principios de diciembre compro otro de merluza, que enviaría a Valencia. Permaneció en Málaga hasta finales de febrero, cuando con una embarcación tomó nuevamente camino de Almería con seda. En esta ciudad, a lo largo de marzo, compró más seda que expedía a Valencia en una galeota consignada a Guerau Bellviure el 24 de marzo. Nuevamente, el 8 de abril, montó en su mula y se dirigió a Málaga, donde habra invertido dinero en amueblar una tienda y la casa contigua. Aqui, y hasta el verano de 1421, momento en que perdemos su rastro, compro cera y seda, que enviaba el 21 de mayo con una nave veneciana a Francesc Marti y Guerau Bellviure. Una red de factores consolidada permite ahorrar esfuerzos; las mercanclas, concentradas por el operador, esperan pacientemente la llegada de las naves.

En el caso de las grandes líneas, la necesidad, quizás de acortar el camino, evitando atraques en mercados innecesarios, quizás de evitar perder tiempo en una corriente contraria, se evidenciaba un nexo entre las embarcaciones de pequeño cabotaje y las grandes naves que relacionaba distintas plazas. Podemos citar el caso de la exportación de frutos secos desde Jávea a Flandes. Habra mercaderes de la ciudad de Valencia que recurrían a comerciantes locales de las poblaciones de la Marina, cuyos puertos, Denia y Jávea, eran fondeaderos permanentes de las naves atlánticas, auxiliares por tanto encargados de estibar aquellos productos agrarios, y otros que debran contratar fletes de pequeñas barcas que transportasen desde Valencia a aquellos puertos los frutos secos que habran ido almacenando meses atrás en sus alfondechs y tiendas de la ciudad ${ }^{41}$. También en el caso de los cargamentos más especializados, los navios portadores de sal o de cereal, como los que recalaban en los cargadores del sur de Sicilia, el patrón tomaba pronto la alta mar recta via de bot hacia el puerto de destino. Las naves fletadas por los mercaderes valencianos con destino a Flandes recalaban en el trayecto sólo en aquellos cargadores del reino, de Denia

\footnotetext{
${ }^{41}$ Enrique CRUSEUES, Denia en el sistema de intercambios bajomedieval, all Congres d'Estudis de la Marina Alta», Dénia, 1990, pp. 171-178.
} 
a Alicante, para después ir directamente a su puerto de destino $0^{42}$. En escasas ocasiones recalaban en puertos como Málaga, Sevilla o Cádiz ${ }^{43}$.

Fuera de estos casos especificos, predominaba la navegación de cabotaje, la cual permitfa rentabilizar los costes del transporte marítimo: "La travesía...era, de puerto en puerto, una larga sucesión de ventas, compras y trueques, entrelazados en un complicado circuito. Y, en el intervalo, el cargamento cambiaba a menudo de naturaleza..." 44 . Práctica que conllevaba emplear más tiempo en estar barado frente a un puerto o una playa. que navegando. De los más de 250 dras que necesitó la galera genovesa Sant'Antonio en 1382 para realizar un periplo por el Mediterráneo oriental, algo más del 60 por ciento del tiempo del viaje estuvo fondeada en los puertos, esperando que los comerciantes finalizasen sus negocios (incluyendo casi más de un mes seguido en Famagusta y 41 dras seguidos en Alejandría $)^{45}$. Del mismo modo, dos siglos más tarde, las galeras venecianas que navegaban por aquellos mares seguran empleando entre el 60 al 70 por ciento del tiempo total de duración del viaje en estar atracadas frente a los puertos, bien porque esperaban la finalización de los intercambios, bien por reparaciones, bien por el mal tiempo ${ }^{46}$.

En un viaje como el de Valencia a Pisa, donde se trasportaba lana, se podía emplear más de 20 días en cargar los vellones en las playas de Tortosa, mientras una escala en Barcelona costaba seis dras más ${ }^{47}$. Evidentemente se trata de días utils o fainers, como dice la documentación, es decir, dras en que se podfa trabajar. Cualquier otro contrato de fletamiento de la ruta Valencia-Flandes remarca esta relación entre tiempo de navegación y de espera. Si tenemos en cuenta que la embarcación hacía las escalas entre puertos muy cercanos de la costa valenciana, para después

\footnotetext{
42 1413, septiembre 6 (ARV. Protocolos, $n^{0}$ 2414); 1415, febrero 27 (Idem, $n^{0}$ 2416); 1416, julio 15 (Idem, $\mathrm{n}^{\circ} 2417$ ); 1417, septiembre 30 y diciembre 29 (Idem, $\mathrm{n}^{0} 2418$ ); 1420, septiembre 5 (Idem, $\mathrm{n}^{\circ} 2421$ ); 1435, mayo 16 (Idem, $\mathrm{n}^{\circ}$ 2432); 1437, mayo 7 (Idem, $\mathrm{n}^{\circ}$ 2774)

${ }^{43} 1419$, agosto 28 (Idem, $n^{0} 2420$ ); 1421, enero 23 (Idem, $\left.n^{0} 2726\right) ; 1439$, marzo 20 (Idem, $n^{\circ} 2435$ ).

${ }^{4}$ Femand BRAUDEL, ob. cil., p. 138.

45John DAY, Prix agricoles en Méditerranée à la fin du XIV siècle (1382), «Annales E.S.C." (1961), I, pp. 629-656. 279-296.

${ }^{46}$ Elena FaSANO-GUARINI, Au XVF siècle: comment naviguent les galères, bidem, pp.

${ }^{47} 1433$, junio 10 (ARV, Protocolos, $n^{0} 2733$ ).
} 
navegar directamente (recta via de bot) hasta Flandes, la proporción no variaba tanto. La nave de Joan Bertran, empleó 32 días para cargar mercancias entre Valencia y Alicante, antes de dirigirse hacia Flandes ${ }^{48}$. En los grandes periplos norte-africano y sardo-siciliano, la dilatación del tiempo de negociación es aún más amplia. La nave castellana de Ginés Gonzálvez, fletada por Francesc Pellicer, zarpo hacia Palermo. En esta ciudad el patrón debra recibir información de su destino: o Nápoles, o Mesina, Siracusa y un cargador de trigo siciliano, entre Catania y Licata. Desde uno de estos lugares retornaría a aguas valencianas ${ }^{49}$. En total, un mes de estancia, barada la nave entre los puertos ${ }^{50}$. Podra darse el caso de periplos más breves ${ }^{51}$, pero lo que es interesante remarcar es que era posible que también en algunos circuitos la embarcación tardase cerca de 50 dras en cargar y descargar las mercancías, en esperar a que los mercaderes cerrasen sus negocios en cada puerto donde fondeaba, lo que significa un periplo total cercano o superior a los dos meses y medio. Es lo mismo que el trayecto se realizase cruzando el Mediterráneo central que bordeando la costa mediterránea de Europa. En el primer caso, la barca de Blasco Gonzálvez tenfa previsto recalar durante $47 \mathrm{días}^{52}$; en el segundo, la nave de Francesc Manlleu, $48^{53}$.

La circulacion de mercancías en los mercados granadinos o norteafricanos se realizaba con viajes más lentos, quizás debido a una más endeble estructura organizativa de las empresas. Recalar en una gran plaza de la costa de Berbería significaba una estancia cercana a los diez días. Asi, los

${ }^{48} 1437$, mayo 7 (Idem, $n^{0}$ 2774). Tiempo parecido requerían las naves de Juan García de Ancorita (1413, septiembre 6. Idein, $\left.\mathrm{n}^{\circ} 2414\right)$ y de Pedro Juanyes (1435, mayo 16. Idem, $\mathrm{n}^{\circ}$ 2432).

${ }^{49} 1416$, junio 25 (Idem, no 2417).

${ }^{50}$ Otras naves, como las de Francesc Giner, Alfonso Rodríguez o Iñigo del Puerto, fletadas por mercaderes valencianos, empleaban como mínimo un tiempo similar en realizar aquel trayecto que tenía por escalas los puertos de las islas catalanas del Mediterráneo central: 1417, septiembre 2 y octubre 15 (Idem, $\left.n^{0} 2418\right) ; 1426$, enero 12 (Idem, $\left.n^{\circ} 2424\right)$.

${ }^{51}$ La nave de Tristan Fortuny contratada por Martí Andreu empleaba unos 20 días en total, contando diez en la descarga de mercancías en Palermo y cuatro entre los cargadores de trigo de Tèrmini o Licata (1431, agosto 14. Idem, $\mathrm{n}^{\circ} 2428$ ).

${ }^{32} 1420$, febrero 1. Idem, $\mathrm{n}^{\circ} 2421$.

${ }^{53}$ La nave partía de Mallorca (donde había recalado durante 6 días), hacía escalas en Ibiza ("e llà stant tant fins la sia dat compliment de sal o altres robes"), Valencia (8), Barcelona (5), Collioure o Génova (5), Piombino o Talamone (8), Gaeta o Nápoles. El mercader tenía opción de retomar tocando en las mismas escalas o de volver a Palermo, desde donde regresaría a Valencia (1448, marzo 23. Idem, $n^{\circ} 801$. M. Doto). 
viajes directos de Valencia a Orán, Mostaganem o Honein costaba ya un mínimo de medio mes, tiempo al que ha de añadirse la duración estricta de la navegación $\mathrm{n}^{54}$. Bajo estas circunstancias, los trayectos con muchas escalas empleaban más tiempo que los viajes a Cerdeña o Sicilia, en algunos casos con una duración cercana a los dos meses sólo en días de no actividad marítima ${ }^{5 s}$. Bastante más tiempo que el que se observa para rutas más lejanas, como el trayecto a Cádiz y Sevilla, o el viaje al Mediterráneo oriental. En un fletamiento de su nave realizado por Juan Fernández de Deva a los comerciantes Joan Ferrer y Joan Arnau, se determinaban 39 días para las labores de estiba en un viaje con destino a Cádiz y retorno, por lo que el trayecto debía acercarse a los dos meses. Viajar al lado opuesto del Mediterráneo costaba más tiempo, sin embargo, se empleaba menos en las numerosas escalas. Por lo menos eso deja intuir el único contrato de flete que refleja el precio. La nave de Martín Pérez de Gamboa fue fletada por Francesc Pellicer para transportar mercancias hasta los puertos del mar Egeo, en un trayecto que contemplaba 43 dras de permanencia en varias escalas del viaje (Mesina, Candia, Qúos, Rodas) ${ }^{56}$. El fletamiento realizado por Tristan Joan de la galera Santa Maria de la Misericordia y Sant Andreu, aceptado por los parçoners, a Antoni Alago, fijaba una duración temporal mínima de cuatro meses, si bien el contrato no llega a decantarse sobre la posibilidad de realizar el viaje a Levante o Poniente. De todas maneras, el patrón daba licencia al mercader para "que puxa carregar e descarregar llà on ben vist li sia e fer port o ports en tots aquells lochs que benvist li sera, anat, vinent e stant a tota sa volentat per salvament de la galera e gents e robes". En 1443, Gracià de Monsoriu, miles, señor y patrón de una galera grosa de mercaderia llamada Santa Maria y

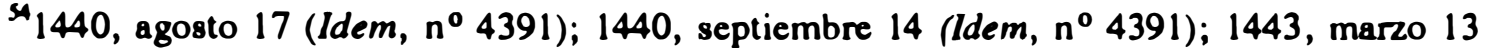
(Idem, $n^{\circ}$ 796. M. Doto).

${ }^{5 s}$ En un contrato el ballener de Juan Sanz Achega debía recalar cerca de 35 días en diferentes puertos de la Mancha mediterrénea (1440, mayo 9. Idem, $n^{\circ} 4391$ ). Eso significa que con el tiempo de navegación, emplearía cerca de dos meses en recorrer las costas valenciana y africana. La nave de Gil Rodríguez empleaba 54 días en la espera del estibado de mercancías, en un trayecto mucho más lejano, que recorría toda la costa africana hasta Túnez (1416, marzo 26. Idem, $n^{0}$ 2417). Días antes el patrón dienense Antoni Ruxot se había comprometido a realizar un viaje por aquella costa, en el que también se contemplaba un máximo de 52 días para comerciar en las diferentes escalas (1416, marzo 24. Ibidem). Del mismo modo, la nave de Pedro Sanz de Becedo debía reservar unos 50 días en atraques (1441, junio 12. Idem, $\mathbf{n}^{0}$ 2411). Meses antes, la contratación del ballener de Femando de Ribadavia al mercader Bemat Rocafort contemplaba 61 días para estibar mercancías (1441, febrero 22. Ibidern).
}

${ }^{56} 1421$, mayo 2 (Idem, $n^{0} 2726$ ); 1418, octubre 6 (Idem, $n^{0} 2419$ ). 
Sant Jordi realizó un contrato de navegación en conserva con los propietarios y el patrón, Lluís Blanch, de otra gran galera mercantil valenciana, la Santa Maria, Sant Jordi y Sant Cristofol ${ }^{57}$. Las rutas fijadas para estas grandes embarcaciones, cada una con una tripulación de 150 remeros, a parte de otros marineros y hombres que realizaban funciones distintas, era la siguiente. A mediados de febrero, como más tarde, la galera de Monsoriu debra zarpar de Valencia en dirección a Barcelona, donde recalaría durante cinco días. Después fard la via de Palerm, donde permanecería otros seis días, antes de continuar rumbo hacia Túnez. En esta ciudad debra esperar la llegada de la otra galera. Ésta partiría más tarde, en dirección a Mallorca, donde atracaría durante tres días per levar les robes que trobard aqui. Acabada la estiba tomaría la via de Alger, donde recalaría durante tres días, y la de Bujia, donde debia permanecer dos días, hasta llegar a Túnez, donde junto a la embarcación compañera sperarant los mercaders qui carregar volran quinze jorns. Por tanto, hasta que ambas naves volviesen a zarpar no sería ya más que finales de marzo. Llegado el momento debian hacer la via de Tripoli, donde atracaría durante cuatro dias, Rodas (tres días) y, finalmente, Alejandría, destino final, donde las galeras debian atracar "per fer port per descarregar e sperar los mercaders quaranta-cinch jorns descarregant e carregant". De Alejandría retornarian a Rodas y, desde aqui, cada una regresarfa por donde vino o según las obligaciones a que se hubiese sometido el patrón con los mercaderes que habran cargado mercanclas. Es decir, que sólo en el tiempo de estancias en las escalas que sirven para recorrer todo el Mediterráneo, ambas galeras, en un itinerario de ida y retorno, tardaron tres meses. Con el tiempo de navegación, cumpliría con seguridad el cuarto.

Todos estos ejemplos demuestran dos elementos claves como son el predominio de la navegación de cabotaje y de la consecuente lentitud del transporte. Sistema de navegación ligado a la endeble estructura de las empresas comerciales, reconocible en la escasa capitalización de los negocios, su elevada fragmentación y otros comportamientos derivados del excesivo respeto que estos hombres de negocios tenfan por un medio natural y económico que no dominaban. Por tanto, la documentación notarial refleja una realidad distinta de la que se podría deducir de las innovaciones técnicas navieras difundidas en esta época. Construir buques de mayor

${ }^{57} 1443$, enero 8 (Idem, no 796, M. Doto). 
tamaño no se tradujo en un crecimiento de la dimensión de los negocios mercantiles. Por el contrario, conllevó una reducción de los costes del transporte, transformación cuyas consecuencias aún no han sido plenamente analizadas, pero que, como defendía F. Braudel, pudo redundar en la disminución del número de navíos de gran tonelaje desde mediados del $\mathrm{XV}$. Las empresas comerciales de la época siguieron recurriendo a un medio de transporte adecuado a sus necesidades y sus recursos.

\section{RÉSUMÉ}

Le développement du commerce du bas moyen âge supposa la diffusion des progrès techniques dans le cadre du transport maritime. Valencie, un marché en expansion à cette époque, subit aussi des transformations fondamentales. Néanmoins, l'analyse des structures et des attitudes des compagnies marchandes oblige à nuancer ces transformations. La prééminence d'un faible financement des affaires et d'une stratégie fragmentaire des activités, se combina avec la prédominance d'une navigation de cabotage, posée, conditionnée par un très grand nombre d'échelles et l'augmentation des transactions.

\section{SUMMARY}

The increase in late medieval trade favoured the spread of technical developments in sea transport. Valencia, a growing market at this period, also went through significant changes. Closer analysis of the structure and behaviour of trading companies allows us to explore this. Persistence of poor financial resources and a piecemeal strategy in trade, combined with use of local shipping, affected the high number of stops and slowness in transactions. 